\title{
Adaptive wideband equalization for frequency dispersion correction in HF band considering variations in interference characteristics and ionosphere parameters
}

\author{
Dmitry Ivanov, Vladimir Ivanov, Vladimir Ovchinnikov ${ }^{*}$, Aleksey Elsukov, \\ and Maria Ryabova \\ Volga state university of technology, 424000, Yoshkar-Ola, Russian Federation
}

\begin{abstract}
Paper presents the outcomes of the studies into the adaptive wideband equalization for frequency dispersion correction in HF band considering variations in interference characteristics and ionosphere parameters. The subject matter of the research were megahertz-bandwidth channels with single-hop F layer propagation mode. There are presented data on variations in the channel amplitude frequency response that are caused by the interference of magneto-ionic components (intramodal multipath). Test facility for carrying out full-scale experiments was developed with the use of Universal Software Radio Peripheral platform supported by the groundbreaking software-defined radio technology. Verification of the developed methods and algorithms was performed in the experiments on oblique sounding over the Cyprus-to-Yoshkar-Ola propagation path by the linearly frequency modulated continuous wave signal.
\end{abstract}

\section{Introduction}

Ionospheric radio channel characteristics significantly vary over slow time due to the influence of propagation medium. These variations can disrupt operation of wideband radio systems and corrupt information that is conveyed by signals. There are several negative factors that influence propagation of radio signals throughout the ionosphere corrupting their amplitude frequency response (AFR) and phase frequency response (PFR). The most harmful effects are intermodal multipath when multiple propagation modes exist, intramodal multipath due to the magneto-ionic splitting, frequency dispersion due to the dependence of the phase velocity of the wave propagation on its frequency and timevarying properties of the ionosphere that cause corresponding variations in the characteristics of radio channels [1-4].

Adaptive equalization for frequency dispersion allows to significantly increase the signal bandwidth. Spreading the bandwidth provides the opportunity to radically solve the problem of stealthiness and noise immunity of HF communication.

\footnotetext{
*Corresponding author: OvchinnikovVV@,volgatech.net
} 
Currently, in many communication systems, correction is based on measuring complexvalued channel frequency response, that is used for computing equalizer correction coefficients. Equalization is carried out by multiplying the received signal subcarriers by the corresponding equalizer coefficients. However, for HF communication systems, this procedure poses a challenge because the parameters of the propagation channel vary depending on the frequency and slow time with a stationarity period of roughly 10 seconds [3]. Furthermore, the usage of ultra-wideband (1 MHz and higher) signals is impossible without devices for equalizing channel frequency response (FR) that is corrupted due to the influence of frequency dispersion. It requires developing methods and algorithms of channel equalization with training mode to update data on varying channel parameters. Data on the complex-valued FR of the wideband channel should be obtained by means of sounding by a test signal. Thus, developing and studying the method of adaptive equalization of channel FR with the possibility of its integration with the state-of-the-art HF communication systems by means of software-defined radio (SDR) technology are topical scientific and technical issues.

The aim of the research was to develop method and algorithm of adaptive equalization for frequency dispersion and interference whitening with training mode in wideband (1 $\mathrm{MHz}$ ) HF communication radio channels with the use of data on channel sounding by linearly frequency modulated continuous wave (LFMCW) signal and SDR technology.

\section{Theoretical fundamentals of channel adaptive equalization}

Currently, the main approach to solving the problems of radio wave propagation is an equivalence principle. According to that principle radio channel can be modeled by an equivalent linear system [4-7] and its associated frequency response (FR) $H(j \omega)$ and impulse response (IR) $h(\tau)$. Let us consider a time-varying radio channel that has the following FR and IR :

$$
\begin{gathered}
H(j \omega, t)=\left\{\begin{array}{l}
H(\omega, t) \cdot \exp [-j \varphi(\omega, t)], \quad \text { if } \quad \omega \in\left[\omega_{c}-\Omega_{c h} / 2, \omega_{c}+\Omega_{c h} / 2\right] \\
0, \quad \text { if } \quad \omega \notin\left[\omega_{c}-\Omega_{c h} / 2, \omega_{c}+\Omega_{c h} / 2\right]
\end{array}\right. \\
h(\tau)=\frac{1}{2 \pi} \int_{-\infty}^{+\infty} H(\omega) \exp [-j \varphi(\omega)] \exp [j \omega \tau] d \omega
\end{gathered}
$$

where $\omega$ - angular frequency, $\omega_{c}$ - mid-band (operating) channel frequency, $\Omega_{c h}$ channel bandwidth, $H(\omega, t)$ - AFR and $\varphi(\omega, t)$ - PFR, $t$ - slow geophysical time, $\tau$ - fast time (delay).

If that model is valid, the signal spectrum $U_{R}(j \omega)$ at the channel output equals the transmitted signal spectrum $U_{T}(j \omega)$ times the channel FR $H(j \omega)$ : $U_{R}(j \omega)=U_{T}(j \omega) \cdot H(j \omega)$ [4]. However, narrowband interferences and fluctuating noise of different origin dominate in the HF band $[8,9]$. Therefore, we assumed that the complex amplitude spectrum of the received signal and interferences is as follows:

$$
Y_{R}(j \omega)=U_{T}(j \omega) \cdot H(j \omega)+\sum_{k=1}^{k_{0}} N_{k}(j \omega)+N_{0}(j \omega),
$$

where $N_{k}(j \omega)$ - narrowband interference spectrum, $N_{0}(j \omega)$ - quasi-white noise spectrum. 
Thus, for communication systems, it is required to filter channel components and additive interferences and noise in (3). Hence, the correction objective is to build an equalizer that allows to mitigate negative effects in the channel.

Equalization of interferences involves their whitening in the channel by dividing spectrum samples of the received signal and noise mixture by the correction function $K(\omega)$ :

$$
\frac{Y_{R}(j \omega)}{K(\omega)}=\frac{U_{T}(j \omega)}{K(\omega)} H(j \omega)+\frac{\sum_{k=1}^{k_{0}} N_{k}(j \omega)+N_{0}(j \omega)}{K(\omega)} .
$$

Interferences and noise correction function is derived by averaging consecutive sampled power spectra within the channel stationarity time [8] and for low spectral power density of echoes is as follows:

$$
K(\omega) \approx \sqrt{\frac{\left\langle N_{0}^{2}(\omega)\right\rangle}{T_{e}}+\sum_{k=1}^{k_{0}} \frac{\left\langle N_{k}^{2}\right\rangle}{T_{e}} \cdot \delta\left(\omega-\omega_{k}\right)},
$$

where $T_{e}$ - time interval for single spectrum measurement.

Equalizer that compensates for dispersion has the following transfer function that is the inverse of the channel frequency response:

$$
H^{-1}(j \omega)=\frac{1}{H(j \omega)}=\frac{H^{*}(j \omega)}{H^{2}(\omega)},
$$

where $H^{*}(j \omega)$ - complex conjugate of the channel FR.

Application of the correction algorithms in the adaptive mode requires periodic estimating channel parameters and spectral power density of interferences and noise by means of periodic testing the channel. This operation mode is typically referred to as equalizer training. In the training mode, it is appropriate to employ fast sounding (roughly $10 \%$ of the channel stationarity time) to leave $90 \%$ of the stationarity time for the operation of a communication system in a wideband radio channel.

\section{Method of measuring wideband radio channel FR}

FR is measured through the channel testing by a spread spectrum signal that allows its compression in the receiver. Previous studies [4] showed that a compressed signal is related to a channel IR. Procedure involves testing of a frequency-ordered set of adjoint narrowband $\omega_{c h}$ channels that constitute a wideband one. Let us assume that the wideband channel consists of $N$ partial channels. For that channel, FR within the stationarity time (roughly 10 seconds) is as follows:

$$
\begin{gathered}
H(\omega, t) \approx H(\bar{\omega}, t)=\text { const } \\
\varphi(\bar{\omega}+\Omega, t) \approx \varphi(\bar{\omega}, t)+\varphi^{\prime}(\bar{\omega}, t) \cdot \Omega
\end{gathered}
$$

where $\varphi^{\prime}(\bar{\omega}, t)=\tau_{g}(\bar{\omega}, t)$ - group delay that equals phase shift in that case.

For FR in the channel bandwidth $\left[-\Omega_{c h} / 2, \Omega_{c h} / 2\right]$, IR is represented as follows: 


$$
\begin{gathered}
h(\bar{\omega}, t, \tau)=\frac{H(\bar{\omega}, t) \cdot \exp (-j \varphi(\bar{\omega}, t))}{2 \pi} . \\
\int_{-\Omega_{c h} / 2}^{\Omega_{c h} / 2} \exp \left[j \Omega\left(\tau-\varphi^{\prime}(\bar{\omega}, t)\right)\right] d \Omega=H(j \bar{\omega}, t) \cdot \frac{\Omega_{c h}}{2 \pi} \sin c\left[\Omega_{c h}\left(\tau-\varphi^{\prime}(\bar{\omega}, t)\right) / 2\right]
\end{gathered}
$$

It is seen that for a random narrowband channel, IR maximum value $\max \left|h\left(\bar{\omega}_{k}, \tau\right)\right|$ yields complex-valued FR sample at the mid-band channel frequency $\omega=\bar{\omega}_{k}$ :

$$
h\left(\bar{\omega}, t, \tau=\varphi^{\prime}(\bar{\omega})\right)=H(j \bar{\omega}, t) \cdot \frac{\Omega_{c h}}{2 \pi}=k_{0} \cdot H(j \bar{\omega}, t) .
$$

Thus, according to the proposed method, FR samples of adjoint narrowband channels are used to compute complex-valued envelope of the narrowband channel IR that yields samples of the complex-valued wideband channel FR. Next, wideband FR samples are used to build wideband channel equalizer that implements inverse filtering.

The most favorable conditions to the application of inverse filtering are ensured in the channels with one-hop propagation mode because in that case intermode multipath fading will not occur. Typically, long distance radio paths exhibit a fairly wide band where only a single mode propagates [10].

Figure 1 presents recorded ionogram of panoramic oblique sounding over the Cyprusto-Yoshkar-Ola radio path by the LFMCW signal and AFR of the wideband (1 $\mathrm{MHz})$ channel from the single mode propagation (SMP) band that was over $5 \mathrm{MHz}$.
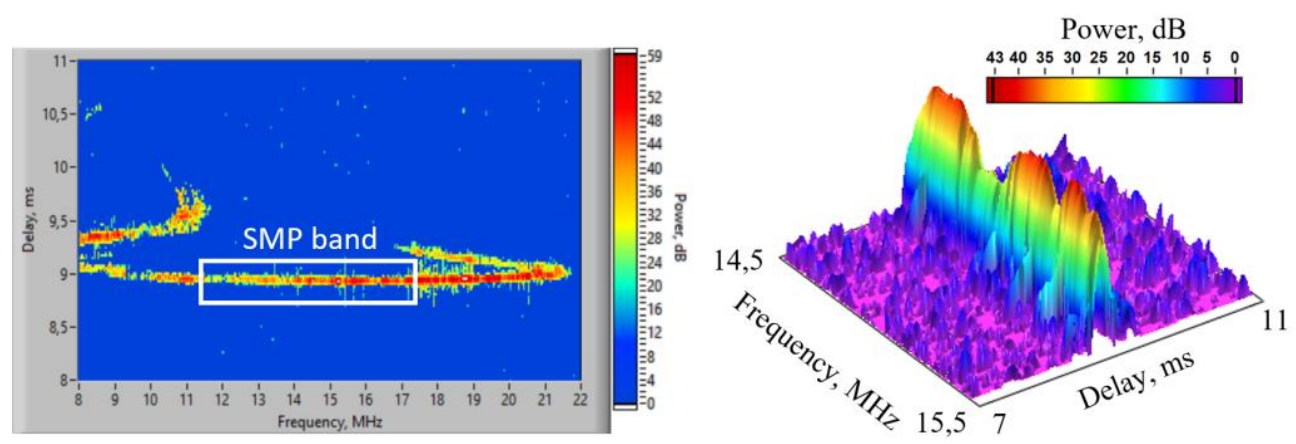

Fig. 1. Ionogram (left) of panoramic oblique sounding over the Cyprus-to-Yoshkar-Ola radio path by the LFMCW signal and AFR (right) of the wideband $(1 \mathrm{MHz}$ ) channel from the single mode propagation band.

It can be seen that AFR exhibits variations of the spectral power density of up to $20 \mathrm{~dB}$ over the bandwidth scale $250-350 \mathrm{kHz}$ due to the interference of ordinary and extraordinary magneto-ionic components. Amplitude correction is applied to mitigate signal selective fading.

\section{Experimental technique and research findings}

Verification of the developed correction algorithm and relevant software that were implemented on the USRP N210 platform was carried out in full-scale experiments. USRP platform is supported by the software-defined radio technology. Wideband (with $1 \mathrm{MHz}$ bandwidth) channels were selected in the SMP band that was determined by real-time data on panoramic sounding over the entire HF band. Chirp rate of LFMCW signal was 100 $\mathrm{kHz} / \mathrm{s}$. Received signal is compressed in the time domain. Compression yields a beat-note 
continuous signal that is divided into shorter adjacent segments of duration $T_{a}=0.08$ for stretch processing. It gives instantaneous complex-valued power delay profiles of channel impulse responses with bandwidth $8 \mathrm{kHz}$ and time resolution of $125 \mu \mathrm{s}$. Complex-valued sample related to the global maximum of channel IR yielded complex-valued FR sample and its complex conjugate $H^{*}(j \omega)$, that was used in the algorithm of correction for dispersion in the channel with $1 \mathrm{MHz}$ bandwidth.

Findings showed that channel characteristics vary over slow time. Thus, in the training mode, it is crucial to periodically update data on the AFR and PFR of the wideband radio channel to adaptively change equalizer coefficients. In the other words, equalizer should adapt to the channel conditions.

Figure 2 presents unequalized and equalized IR of the wideband radio channel with 1 $\mathrm{MHz}$ bandwidth on the Cyprus-to-Yoshkar-Ola propagation path.

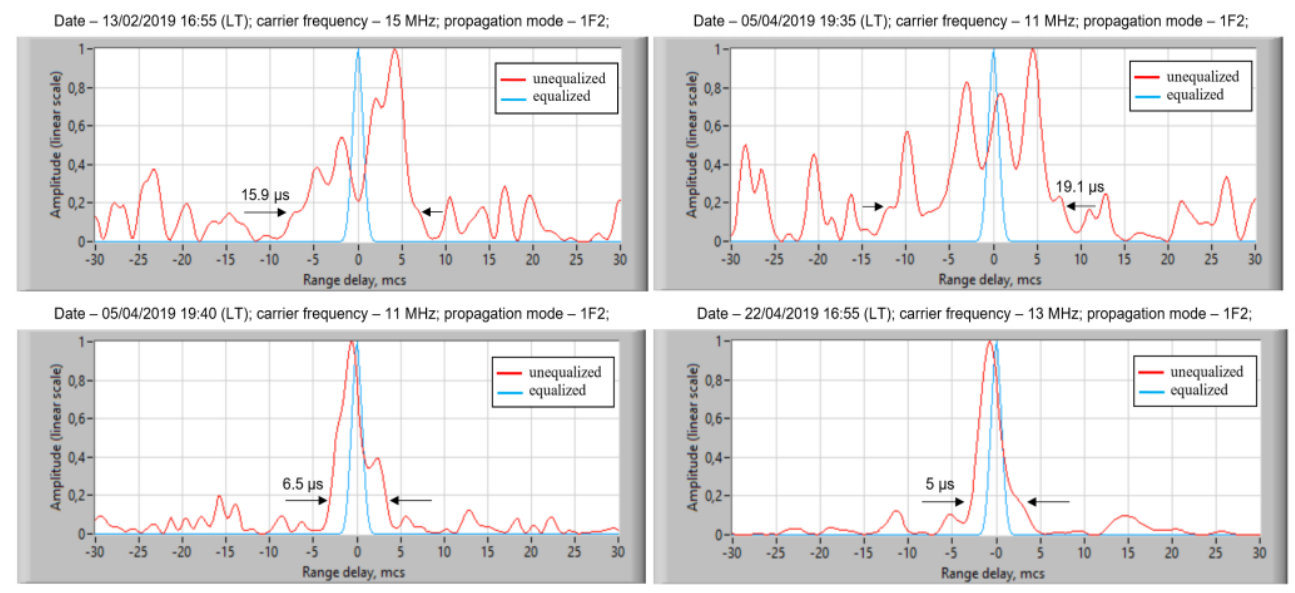

Fig. 2. Unequalized and equalized IR of 1-MHz channel.

It is seen that before correction IR main lobe had a duration from roughly $5 \mu$ s to $19 \mu \mathrm{s}$, instead of $1 \mu \mathrm{s}$. The effect is caused by the frequency dispersion that results in a delay spread in the wideband channel. Findings showed that adaptive equalization by the method of inverse filtering ensures a decrease in the IR main lobe duration to the theoretical values (approximately $1 \mu \mathrm{s}$ ).

\section{Conclusions}

Developed methods and algorithms of adaptive correction for frequency dispersion were verified in the full-scale experiments on sounding over Cyprus-to-Yoshkar-Ola propagation path by the LFMCW signal. It was shown that AFR of the channel in the SMP band exhibited significant (up to $20 \mathrm{~dB}$ ) variations in the spectral power density over the bandwidth scale of $250-350 \mathrm{kHz}$ due to the interference of ordinary and extraordinary magneto-ionic components. Measured wideband channel IR suffered from spreading by up to 5-19 times due to the influence of frequency dispersion. Findings showed that adaptive equalization by the method of inverse filtering corrects for frequency dispersion. Furthermore, it is appropriate to employ fast sounding (roughly $10 \%$ of the channel stationarity time) to leave $90 \%$ of the stationarity time for the operation of a communication system in a wideband radio channel.

This work was supported by the grant No. 18-19-00401 from the Russian Science Foundation. 


\section{References}

1. R. P. Basler, G. H. Price, R. T. Tsunoda, T. L. Wong, Ionospheric distortion of HF signals, Radio Science, 23(4), pp. 569-579. https://doi.org/10.1029/RS023i004p00569 (1988)

2. K. Khoder, R. Fleury, P. Pagani, Monitoring of ionosphere propagation conditions using opportunistic HF signals, in The 8th European Conference on Antennas and Propagation (EuCAP 2014), The Hague, pp. 2697-2701. https://doi.org/10.1109/ EuCAP.2014.6902381 (2014)

3. S. Dhar, B. D. Perry, Equalized Megahertz-Bandwidth HF Channels for Spread Spectrum Communications, MILCOM 1982 - IEEE Military Communications Conference - Progress in Spread Spectrum Communications, Boston, MA, USA, pp. 29.5-1-29.5-5. https://doi.org/10.1109/MILCOM.1982.4805973 (1982)

4. V. A. Ivanov, D. V. Ivanov, N. V. Ryabova, M. I. Ryabova, A. A. Chernov, V. V. Ovchinnikov, Studying the parameters of frequency dispersion for radio links of different length using software-defined radio based sounding system, Radio Science, 54(1), pp. 34-43. https://doi.org/10.1029/2018RS006636 (2019)

5. D. V. Ivanov, V. A. Ivanov, N. V. Ryabova, M. I. Ryabova, A. A. Kislitsin, V. V. Ovchinnikov, Studying frequency dispersion in transionospheric radio paths using the estimates of the total electron content, 12th European Conference on Antennas and Propagation, EuCAP 2018; London; United Kingdom; 9-13 April 2018. Vol. 2018, Issue CP741, pp. 1-5. https://doi.org/10.1049/cp.2018.0474

6. V. A. Ivanov, D. V. Ivanov, N. V. Ryabova, M. I. Ryabova, A. A. Chernov, V. V. Ovchinnikov, Studying the parameters of frequency dispersion for radio links of different length using SDR based sounding system, 2017 XXXIInd General Assembly and Scientific Symposium of the International Union of Radio Science (URSI GASS), Montreal, QC, pp. 1-3. https://doi.org/10.23919/URSIGASS.2017.8105045 (2017)

7. D. V. Ivanov, V. A. Ivanov, N. N. Mikheeva, N. V. Ryabova, M. I. Ryabova, Propagation of broadband HF signals in a medium with nonlinear dispersion, Journal of Communications Technology and Electronics, 60 (11), pp. 1205-1214. https://doi.org/10.1134/S1064226915110066 (2015)

8. V. A. Ivanov, D. V. Ivanov, N. V. Ryabova, M. I. Ryabova, A. A. Chernov, A. A. Elsukov, Algorithms and software of whitening of noise in the reception of broadband chirp signals in the HF communication channel, Journal of Applied Engineering Science, 13(4), pp. 245-250. https://doi.org/10.5937/jaes13-9342 (2015)

9. V. V. Ovchinnikov, N. V. Ryabova, A. A. Elsukov, Adaptive HF Signal Detection Algorithm CFAR and Its Verification By Means of SDR Based Digital Ionosonde with USRP Platform, 2018 Systems of Signal Synchronization, Generating and Processing in Telecommunications (SYNCHROINFO), Minsk, pp. 1-5. https://doi.org/10.1109/ SYNCHROINFO.2018.8456970 (2018)

10. V. V. Ovchinnikov, V. A. Ivanov, N. V. Ryabova, Effect of Season on Single Mode Propagation Band of Short-Wave Signals, 2019 Russian Open Conference on Radio Wave Propagation (RWP), Kazan, Russia, pp. 325-328. https://doi.org/10.1109/ RWP.2019.8810376 (2019) 